\title{
Diseño de un sistema de información integral para el Archivo del Seminario Metropolitano San Atón de Badajoz
}

Designing an integrated information system for the archive of the San Aton Metropolitan Seminary of Badajoz (Spain)

María Guadalupe PÉrez ORTIZ, Francisco GonZÁlez LozANo

Seminario Metropolitano de san Atón. 06006 Badajoz, mgperort@alcazaba.unex.es, fglozano@hotmail.com

\begin{abstract}
Resumen
El archivo del Seminario Metropolitano San Atón de Badajoz custodia la documentación generada y recibida por esta entidad en el transcurso de su dilatada historia, la cual se remonta a mediados del siglo XVII. La labor educativa y pedagógica desarrollada por el Seminario fue crucial para la historia educativa de Extremadura en los siglo XVIII-XIX. En base a la importancia de la entidad se vienen desarrollando diversas tareas para la preservación y difusión de los fondos contenidos en su biblioteca y archivo. Se presenta el un sistema de información integral compuesto de tres pilares que se ha desarrollado para preservar y difundir sus fondos: el catálogo, el tesauro y el gestor documental, que forman un grupo unido que tendrá como fin principal la recuperación y difusión de la documentación contenida en este archivo.

Palabras clave: Archivos eclesiásticos. Archivos de seminarios. Seminario Metropolitano San Atón (Badajoz, España). Sistemas de información integral. Catálogo. Tesauro. Cuadro de clasificación. Gestores documentales.
\end{abstract}

\section{Introducción: aproximación al Archivo y trabajo previo}

Después de más de dos años de trabajo en el Archivo del Seminario de San Atón en los que hemos desarrollado tareas de inventariado y clasificación de sus fondos desarrollando un completo inventario y un cuadro de clasificación aplicable al conjunto de su documentación trabajo expuesto en el XIX Encuentro Internacional sobre Sistemas de Información y Documentación (Pérez Ortiz; González Lozano, 2014)_, nos centramos ahora en el desarrollo, todavía en su fase inicial, de un completo sistema de información para el archivo.

Antes de comenzar con estas nuevas cuestiones recordaremos muy brevemente la historia de nuestro seminario, archivo y del cuadro de clasificación diseñado para la entidad dado que nos permitirán conocer la institución y los procesos archivísticos que se han desarrollado en

\begin{abstract}
The archive of the Metropolitan Seminary San Aton of Badajoz custodies the documentation generated and received by this entity in the course of its long history, which goes back in the mid-17th century. The educational and pedagogical work developed by the Seminary of Badajoz was crucial to the educational history of Extremadura in the 18 th and $19^{\text {th }}$ centuries. This paper presents the comprehensive information system devoped for the preservation and disseminations of its holdings, which consist of three pillars: the catalogue, the thesaurus and the document management system.
\end{abstract}

Keywords: Ecclesiastical archives. Catholic seminar archives. Metropolitan Seminary San Aton (Badajoz, Spain). Comprehensive information systems. Catalogue. Thesaurus. Classification schema. Automated document management systems.

ella. La Iglesia conocedora del rico patrimonio bibliográfico y documental que atesoran sus bibliotecas y archivos ha demostrado siempre un grandísimo interés por su salvaguarda. En relación a los archivos, en un primer momento se pretendía intemporalizar la doctrina; posteriormente, los archiveros eclesiásticos se encargaron de describir la documentación en ellos conservada. En relación a los seminarios, será a partir del Concilio de Trento (1545-1563) cuando se comiencen a erigir estos centros que surgen para dar una formación integral al clero que hasta la fecha era un tanto escasa. Se iniciaba entonces la trayectoria histórica $y$, por extensión, archivística de estas nuevas instituciones. En el caso de Badajoz se observa la preocupación por la instrucción de los clérigos y los diferentes modos de establecerla, pero la escasez de rentas hizo demorar la creación del Seminario prácticamente un siglo desde la promulgación de los decretos tridentinos. Para su puesta en marcha serán de suma importancia los lega- 
dos y mandas pías que se constituyeron para tal fin. La más importante, la obra pía del canónigo Rodrigo Dosma Delgado, que cedió sus casas para la erección del Seminario, como consta en el Libro de Fundación de la Institución. Se instituye el Seminario Conciliar de San Atón en la ciudad de Badajoz, el 26 de Mayo de 1664, siendo obispo de la diócesis, fray Jerónimo Rodríguez de Valderas (Rubio Merino, 1999, p. 328).

El archivo, carente de una historia particular que analice su institución y desarrollo, se crea a la vez que el Seminario. Contiene la documentación producida y recibida por la entidad en el trascurso de su actividad educativa, litúrgica, pastoral y la derivada de la conservación y gestión de su patrimonio; así como la de otros colegios-seminarios dependientes de él. En un primer momento la documentación se encontraba dispuesta en legajos que fueron adaptados en cajas archivadoras para su mejor conservación. Una vez revisados, la documentación fue cotejada con un catálogo descriptivo previo realizado conforme a las Constituciones de 1664, 1783 y 1849 , respectivamente y al Reglamento de 1863. En base a ello se confeccionó un cuadro de clasificación, adaptado a la ISAD (G). La consulta del fondo es libre y gratuita, restringida en lo referente al derecho de intimidad y honor de las personas por las leyes vigentes.

En líneas generales el fondo del archivo queda constituido de la siguiente forma: Fondo del Seminario Diocesano de San Atón; Fondo del Colegio-Seminario de San Benito (Villanueva de la Serena); Fondo del Colegio-Seminario Nuestra Señora de la Coronada (Villafranca de los Barros); Fondo de los Seminarios de Elvas y Olivenza; Fondo musical; y Fondo colecciones. El cuadro de clasificación diseñado para el archivo es el que se expone en la tabla I.

$\begin{array}{ll}1.0 & \text { Gobierno } \\ 1.01 & \text { Autoridad Pontificia } \\ 1.01 .01 & \text { Breves } \\ 1.02 .02 & \text { Bulas } \\ 1.02 .03 & \text { Sagrada Congregación del Clero } \\ 1.02 & \text { Autoridad Real } \\ 1.02 .01 & \text { Ordenanzas y Provisiones } \\ 1.02 .02 & \text { Reales Cédulas } \\ 1.03 & \text { Documentación Episcopal } \\ 1.03 .01 & \text { Libro Fundación Seminario } \\ 1.03 .02 & \text { Despachos Obispo/Obispado } \\ 1.03 .03 & \text { Decretos Episcopales } \\ 1.03 .04 & \text { Libro Inventario Obispado } \\ 1.03 .05 & \text { Constituciones } \\ 1.03 .06 & \text { Reglamentos } \\ 1.03 .07 & \text { Actas de Visita } \\ 1.03 .08 & \text { Doctrina } \\ 1.04 & \text { Conferencia Episcopal Española } \\ 1.04 .01 & \text { Planes de Formación Seminario Mayor }\end{array}$

Planes de Formación Seminario Menor

Encuentros de Rectores

Rectorado y Claustro Académicos

Actas Junta Consultiva, Disciplina y Hacienda

Actas Academias Literarias

Memorandum

Actas Claustro de Profesores

Actas Formadores

Centro Superior de Estudios Teológicos

Actas Académicas

Informes Biblioteca

Universidad Pontificia de Salamanca

Estatutos

Grados académicos

\section{Secretaría}

(Secretaría Seminario Mayor)

Asuntos Académicos

Solicitud/Justificantes

Matrículas

Actas de Exámenes

Notas

Becas

Expedientes de Conducta

Listado de Alumnos

Asuntos Generales

Certificaciones

Correspondencia

Expedientes Personales

Informaciones

Planes de Estudios Académicos

(Secretaría Seminario Menor)

Asuntos Académicos

Libros de matrículas

Libros de notas

Becas

Asuntos Generales

Correspondencia

Normativas y disposiciones

Administración

Administración General

Cuentas

Obras

Pagos

Recibos/Justificantes

Personal

Nóminas

Seguridad Social

IRPF

Bienes

Censos

Escrituras

Inventario de Bienes

Libro Becerro

Colectas

3.04.01 Estipendios de misa

3.04.02 Campaña Día del Seminario

3.04.03 Donativos

Obras Pías

Administración

Cuentas

Inventario de Bienes

Libro de Cuentas

3.05.02 Obras Pías

Arias de Hoces Bravo de Laguna

Diego Hernández Duque de Béjar

Feria Fonseca

Francisco López Chávez Francisco Mejías 
Josefa Vicente Rino Marques de Lapilla

Pedro Casas Guerrero Rodrigo Dosma

Somoza Ribera

4.0 Órdenes Sagradas

$4.01 \quad$ Expedientes de órdenes

$4.02 \quad$ Vocaciones

5.0 Instituciones Dependientes

5.01 Seminario Menor Elvas-Olivenza

5.01.01 Censos

5.01.02 Correspondencia

5.01.03 Cuentas

5.01.04 Foro de Trigo

5.01.05 Informes

5.02 Seminario Menor Nuestra Señora de la Coronada. Villafranca de los Barros

5.02.01 Cuentas

5.02 .02 Notas

5.03 Colegio-Seminario Menor de San Benito. Villa-
nueva de la Serena

5.03.01. Cuentas

5.03.02 Escrituras

5.03.03 Matrículas

5.03.04 Notas

5.03.05 Solicitudes

6.0 Fondo Musical

7.0 Fondo Colecciones

Tabla I. Cuadro de clasificación

\section{Sistema de información}

En el Diccionario Enciclopédico de Ciencias de la Documentación (López Yepes, 2004, p. 451) se define el término Documentación como "acción y efecto de documentar y de buscar información con el fin de que sirva al usuario...". Por otro lado, así comenzaba el profesor Del Valle (1991, p. 101) un artículo en el que describía la aplicación de la Documentación a la Archivística mediante la utilización de técnicas de indización y lenguajes como el tesauro a instrumentos de información como el catálogo:

La Documentación es la actividad de comunicación humana que se ocupa de los procesos de transferencia de información, desde su generación hasta su uso. Estos procesos incluyen la selección y adquisición, organización, interpretación, almacenamiento, recuperación y difusión de la información contenida en cualquier tipo de soporte.

En este sentido, el catálogo, el tesauro y el gestor documental formarán un grupo unido que tendrá como fin principal la recuperación y difusión de la documentación. Por tanto, los tres elementos que constituirán el sistema de información del Archivo del Seminario (en adelante ASMMB) son el catálogo documental, en él que se presentan las características externas e internas de cada uno de los documentos ubicados en el fondo; el tesauro, esto es el vocabulario destinada a representar de manera unívoca el contenido de los documentos y de las consultas, y a ayudar al usuario en la indización y en la correcta recuperación de la información consultada; y el gestor documental automatizado, que nos permitirá el acceso a la información relativa a cada documento de manera sencilla y rápida, logrando acceder tanto a las características externas como al contenido de los documentos a través de un sencillo interfaz, e incluso en un futuro al documento digitalizado.

\subsection{Catálogo documental}

Uno de los objetivos que nos propusimos fue facilitar a los usuarios y al propio archivo el acceso a la información contenida en nuestra documentación, es decir, acercar al usuario potencial la información por medio de la descripción de documentos (Heredia, 1982, p. 7). El resultado se hace tangible en los llamados instrumentos de descripción. En nuestro caso hemos optamos por la elaboración de un catálogo documental, ya que de este modo el contenido de las agrupaciones documentales podrá ser buscado, localizado y recuperado por medio de un instrumento de información que se encontrará automatizado. La normalización que se ha seguido corresponde con las normas $\operatorname{ISAD}(G)$, aunque algunos de sus elementos no han sido empleados puesto que la documentación no se adaptaba a ellos (ISAD(G), 2000). Los elementos descriptivos que van a componer nuestras fichas son los que se exponen:

\section{Área de mención de identidad:}

Signatura: En el ASMMB existía un sistema de signaturización previa en el archivo, que nosotros hemos repetido para el catálogo. Los elementos que componen la signatura son: código del Archivo: ASMMB; código de la documentación por secciones: Gobierno, Secretaría, Administración, etc.; número arábigo de instalación del volumen: Libro 1, 2, 3..., Caja 1, 2, 3... y número de agrupación documental dentro de la unidad de instalación: $1,2,3 \ldots$

ASMMB Sección Gobierno, caja 3, 1.

Fecha y lugar de producción: Se compone de: Data crónica expresada como una fecha simple o un periodo cronológico y la data tópica que expresará el lugar en su ortografía actual. Además se tendrá en cuenta, los casos en los que falta alguna indicación del año, mes, día o lugar, la información lograda por otras fuentes va entre corchetes: [1850.Badajoz]; si desconocemos alguno de los datos: s.l. (sin lugar) y s.d. (sin data); si conocemos el siglo: [17--].

1829, marzo, 7. Badajoz 


\section{Área de contenido}

Nota-resumen alcance y contenido: Sirve para identificar la forma y el contenido de la unidad de descripción de manera que los usuarios conozcan la información y juzguen su interés para su posterior consulta.

[Expedientes personales para acceso al Seminario, 1856].

\section{Área de condición de acceso y utilización}

Manifestación manuscrita/impresa: Se especificará en este lugar si se trata de un expediente manuscrito, impreso o combinación de ambas situaciones.

Lengua de los documentos: Se refiere a los datos sobre la lengua o lenguas, tipo de escrituras y sistemas de símbolos utilizados.

Características físicas: Se indicará el número de folios que constituyen el expediente. Si hay algunos folios con distinto tamaño, se hará constar. En Conservación se reflejan las condiciones físicas del expediente: B (buena), R (regular), M (mala). Se indicarán las causas. En llustraciones se hace constar la presencia de ilustraciones o elementos ornamentales. En Soporte se especificará el material siempre que sea diferente al papel (por ejemplo, pergamino).

Manuscrito, 1 expediente (9 folios y 1 folio doble) Latín y castellano. Buena

Área de notas: Se trata del campo relativo a las observaciones en el que consignamos algunas cuestiones que deben saber nuestros usuarios.

En conclusión, la ficha catalográfica diseñada para el ASMMB intentará describir los documentos en tres sentidos: En cuanto a su contenido y localización temporal, resumiendo la información contenida en pocas líneas, a fin de orientar a nuestros usuarios sobre la conveniencia o no de su lectura; en cuanto a sus características formales: volumen, tamaño, soporte, conservación, etc.; y en relación a su localización espacial dentro de nuestro fondo.

El catálogo diseñado para el ASMMB se compondrá de dos partes: las fichas documentales y los índices. Estos últimos serán el topográfico (poblaciones); de materias (diferentes temáticas); onomástico (nombres de personas); de cargos (cargo y persona que lo ostenta); cronológico expone (fechas); y de entidades (instituciones). Mostramos a modo de ejemplo cinco fichas extraídas del catálogo del ASMMB (Tabla II) y ejemplos de sus entradas en los índices (Tablas III a VIII).

\section{ASMMB-1.1-(2)}

5 de junio de 1853-Mérida

Cuestionario de 200 preguntas para obtención título en Teología

Manuscrito, 1 expediente (9 folios y 1 folio doble)

Latín y castellano. Buena

Contiene:

5 de junio de 1853-Mérida. Cuestionario de 200 preguntas para obtención del título en Teología.

1 de abril de 1853-S.L. Directrices para realizar examen de Bachiller en Teología.

ASMMB-1.1-(3)

4 de mayo de 1892-27 de septiembre de 1926-MadridBadajoz

Reglamento para los establecimientos provinciales de beneficencia de la ciudad de Badajoz

Manuscrito, 1 expediente (7 cuartillas y 2 folios)

Castellano. Buena

Contiene:

[Siglo XIX]. Reglamento para los establecimientos provinciales de beneficencia de la ciudad de Badajoz. 4 de mayo de 1892-Madrid. Comunicación ministerio de gobernación sobre cuestiones relativas a la beneficencia en Badajoz.

14 de octubre de 1896-Badajoz. Carta emitida por D. José Comino a la Junta de Beneficencia.

27 de septiembre de 1926-Badajoz. Petición al Rector del Seminario de la Junta provincial de beneficencia de expedientes relativos a obras.

\section{ASMMB-1.1-(4)}

1 de marzo de 1868-Badajoz

Actas del consejo de disciplina formado en Seminario San Atón

Manuscrito, 1 expediente

Castellano. Buena

Contiene:

1 de marzo de 1868-Badajoz. Actas del consejo de disciplina formado en Seminario San Atón.

2 de junio de 1868-Badajoz. Directrices confeccionadas por el Rector del Seminario Nicolás Arnáiz sobre cuestiones de exámenes.

ASMMB-1.1-(5)

[1783-1797]-Badajoz

Constituciones confeccionadas para el Seminario San Atón por el obispo Alonso Solís y Gragera

Manuscrito, 1 expediente

Castellano Buena

ASMMB-1.1-(6)

16 de julio de 1854-Badajoz

Memorandum sobre el Seminario Conciliar de San Atón

elaborado por el rector D. José Quevedo

Manuscrito, 1 expediente

Castellano. Buena

Tabla II. Ejemplos de fichas catalográficas

\begin{tabular}{ll}
\hline Lugar & $N^{\circ}$ doc. \\
\hline Badajoz (Ciudad) & $2,3,5$ \\
\hline Badajoz (Diócesis) & 4 \\
\hline Madrid (Ciudad) & 2 \\
\hline Mérida (Ciudad) & 1 \\
\hline [s.l.] & 1 \\
\hline
\end{tabular}

Tabla III. Índice topográfico 


\begin{tabular}{lc}
\hline Materias & $N^{\circ}$ doc. \\
\hline Beneficencia & 2 \\
\hline Disciplina & 3 \\
\hline Exámenes & 1 \\
\hline Grados & 1 \\
\hline Informaciones & 5 \\
\hline Reglamentos y Constituciones & 2,4 \\
\hline
\end{tabular}

Tabla IV. Índice de materias

\begin{tabular}{lc}
\hline Nombre & No doc. $^{\circ}$ \\
\hline Arnáiz, Nicolás (rector) & 3 \\
\hline Comino, José (rector) & 2 \\
\hline Quevedo, José (Rector) & 5 \\
\hline Solís y Gragera, Alonso (Obispo) & 4 \\
\hline
\end{tabular}

Tabla V. Índice onomástico

\begin{tabular}{lc}
\hline Cargos & $N^{\circ}$ doc. \\
\hline Junta de Beneficencia & \\
\hline Comino, José & 2 \\
\hline Obispo & \\
\hline Solís y Gragera, Alonso & 4 \\
\hline Rector & \\
\hline Arnáiz, Nicolás & 3 \\
\hline Quevedo, José & 5 \\
\hline Comino, José & 2 \\
\hline
\end{tabular}

Tabla VI. Índice de cargos

\begin{tabular}{lc}
\hline Año & $N^{\circ}$ doc. \\
\hline 1783 & 4 \\
\hline 1797 & 4 \\
\hline 1853 & 1 \\
\hline 1854 & 5 \\
\hline 1868 & 3 \\
\hline 1892 & 2 \\
\hline 1926 & 2 \\
\hline [s. XIX] & 2 \\
\hline
\end{tabular}

Tabla VII. Índice cronológico

\begin{tabular}{ll}
\hline Entidad & $N^{\circ}$ doc. \\
\hline Junta de Beneficencia & 2 \\
\hline Consejo de Disciplina. Seminario & 3 \\
\hline Seminario Metropolitano San Atón & $3,4,5$
\end{tabular}

Tabla VIII. Índice de entidades

\subsection{Tesauro}

Entendemos por tesauro "el vocabulario de un lenguaje de indización controlado, organizado formalmente, con objetivo de hacer explícitas las relaciones a priori entre conceptos de los tipos: más genéricos que o más específico que" (Norma ISO UNE 25964-1). Por tanto, sus prin- cipales funciones serán: la normalización del vocabulario eliminando la ambigüedad del lenguaje; la inducción, indicando las alternativas posibles que se pueden utilizar para la recuperación; la representación, estableciendo correspondencia entre los descriptores y los conceptos presentes en el documento; la combinación de elementos en la recuperación; la reunión de descriptores y no descriptores; la localización de los descriptores más apropiados; la univocidad semántica, evitando la presencia de sinonimia y polisemia; y la incorporación de nuevas notas (Slype, 1999, p. 22-30).

El tesauro sobre el que estamos trabajando se compone de una base léxica, que se encuentra estructurada en relaciones jerárquicas, asociativas y de equivalencia. En cuanto a los descriptores utilizados, se pueden clasificar como se indica en la Tabla IX.

\begin{tabular}{|c|c|c|}
\hline Tipo & Descriptor & Ejemplos \\
\hline \multirow[t]{2}{*}{$\begin{array}{l}\text { Por la carga } \\
\text { informativa }\end{array}$} & $\begin{array}{l}\text { Primarios: son los } \\
\text { términos o conjuntos de } \\
\text { términos que representan } \\
\text { un concepto de manera } \\
\text { unívoca, sin posibilidad } \\
\text { alguna de ambigüedad. }\end{array}$ & $\begin{array}{l}\text { Seminario } \\
\text { Educación }\end{array}$ \\
\hline & $\begin{array}{l}\text { Secundarios: } \\
\text { descriptores que } \\
\text { acompañan a otros } \\
\text { descriptores para } \\
\text { restringir la demanda. }\end{array}$ & $\begin{array}{l}\text { Seminario } \\
\text { Metropolitano } \\
\text { San Atón }\end{array}$ \\
\hline \multirow[t]{3}{*}{$\begin{array}{l}\text { Por } \\
\text { cobertura } \\
\text { temática }\end{array}$} & $\begin{array}{l}\text { Onomásticos: } \\
\text { representan el nombre de } \\
\text { una persona o de una } \\
\text { institución. }\end{array}$ & $\begin{array}{l}\text { Tirso Lozano } \\
\text { Obispado de } \\
\text { Badajoz }\end{array}$ \\
\hline & $\begin{array}{l}\text { Geográficos: abarcan } \\
\text { todo tipo de conceptos } \\
\text { vinculados con lugares. }\end{array}$ & $\begin{array}{l}\text { Badajoz } \\
\text { (Ciudad) } \\
\text { Badajoz } \\
\text { (Diócesis) }\end{array}$ \\
\hline & $\begin{array}{l}\text { Temáticos: descriptores } \\
\text { de materia, y son } \\
\text { considerados como los } \\
\text { más importantes dentro } \\
\text { de nuestro tesauro. }\end{array}$ & $\begin{array}{l}\text { Matrículas } \\
\text { Órdenes }\end{array}$ \\
\hline \multirow[t]{2}{*}{$\begin{array}{l}\text { Por } \\
\text { composición }\end{array}$} & $\begin{array}{l}\text { Simples: se trata de } \\
\text { descriptores constituidos } \\
\text { por un solo término. Son } \\
\text { unívocos y primarios. }\end{array}$ & $\begin{array}{l}\text { Rector } \\
\text { Economía }\end{array}$ \\
\hline & $\begin{array}{l}\text { Compuestos: Se refieren } \\
\text { a un concepto utilizando } \\
\text { un sintagma nominal o } \\
\text { preposicional. Se utilizan } \\
\text { para eliminar la } \\
\text { ambigüedad de los } \\
\text { descriptores simples. }\end{array}$ & $\begin{array}{l}\text { Admisiones de } \\
\text { alumnos } \\
\text { Expedientes } \\
\text { personales }\end{array}$ \\
\hline
\end{tabular}

Tabla IX. Clasificación de descriptores en el ASMMB

Sabido es por todos la importancia de utilizar una terminología unívoca para la construcción 
del tesauro superando las posibles sinonimias $y$ polisemias, que, en el caso de la documentación de nuestro archivo, son bastante frecuentes. Veamos cómo hemos solucionado algunos casos:

\section{Polisemia:}

- Paréntesis: Mediante la introducción entre paréntesis de un calificador que concrete el significado del descriptor. Por ejemplo, Badajoz (ciudad) y Badajoz (diócesis)

- Por sintagmatización: se ha transformado el término en un sintagma nominal. Por ejemplo, expedientes personales.

- Por no descriptor: a través de la utilización de un sinónimo. Evidentemente ha sido esta forma la más utilizada. Por ejemplo, prebendas USE becas.

\section{Sinonimia:}

- Por genérico: se han sustituido descriptores demasiado específicos por uno más general. Por ejemplo, reparaciones, reformas, remodelaciones del Seminario se sustituyen por rehabilitación Seminario.

- Por la raíz: varios términos se sustituyen por su sema principal. Por ejemplo, archiveros, Archivística se sustituye por archivos.

La delimitación del área de nuestro tesauro ha sido una tarea sencilla puesto que abarcaba el conjunto de documentos situados en el ASMMB y no una parte de los mismos. A nivel de profundidad, optamos por un grado medio, marcado en la gran mayoría de los casos por el propio carácter documental. Hemos intentado en todo momento que la profundización y la amplitud de cada área o campo fueran lo más homogéneas posibles, aunque debemos reconocer que no siempre ha sido posible; existirán unos campos semánticos más desarrollados que otros, especialmente los que se relacionen con las cuestiones educativas, si tenemos en cuenta que el Seminario de Badajoz pertenece a este grupo de instituciones.

En cuanto a la selección de fuentes: por una parte, la documentación del ASMMB desde 1464 a nuestros días, que como es lógico es la principal. Por otra parte, obras de referencia: enciclopedias, diccionarios técnicos, fuentes bibliográficas y documentales y otros tesauros que nos han permitido observar aspectos aplicables al nuestro.

Una vez seleccionadas las fuentes, procedimos a la extracción de los términos en dos etapas: Primero, se creó de un fichero alfabético, para lo que elaboramos una ficha para cada uno de los pre-descriptores seleccionados, con el nombre del descriptor, faceta o campo de inclusión, nota de alcance, términos equivalentes, término genérico, términos específicos, términos relacionados, y observaciones. En segundo lugar, se procedió a comprobar que los pre-descriptores no poseyeran doble sentido, de esta forma comenzaremos a reducir la lista significativamente.

- Distinguiendo descriptores y no descriptores. Por ejemplo, expedientes personales UP expedientes de alumnos.

- Adjetivos y adverbios aislados son evitados.

- Los infinitivos de los verbos son sustantivados. Por ejemplo, se utiliza administración, no administrar.

- El número: en plural los términos de entidades que pueden ser cuantificados. Por ejemplo, seminarios, obispados, colegios. En singular entidades no contables, nociones abstractas, instituciones singulares. Por ejemplo, Seminario Metropolitano San Atón, Obispado de Badajoz.

Para la distribución de nuestro tesauro hasta el momento, sobre el que aún estamos trabajando, hemos diseñado cinco áreas temáticas:

1. Educación: cuestiones referidas a la amplísima labor educativa y pedagógica que se ha desarrollado en el Seminario de Badajoz desde el siglo XVII. Como es obvio, esta área va a ser más voluminosa que el resto, rompiendo con la homogeneidad que hemos tratado de conferir a esta herramienta en su estructura orgánica.

2. Instituciones civiles: todo lo concerniente a la administración central, local y territorial del Estado en relación al Seminario. Al tratarse de una entidad educativa en la que se expedían títulos académicos homologados, la comunicación con este tipo de instituciones ha sido constante.

3. Instituciones eclesiásticas: todo lo relativo a instituciones que componen la Iglesia y a las funciones que acometen cada una de ellas en relación al Seminario.

4. Órdenes Sagradas: cuestiones relativas a los diferentes procesos para la recepción del Orden sacerdotal.

5. Patrimonio: cuestiones relativas a la economía funcional, censos, mandas pías y rentas.

De la distribución expuesta podemos entender que las familias semánticas enclavadas en el apartado educación, instituciones eclesiásticas y patrimonio, por este orden, serán las más volu- 
minosas en cuanto a descriptores y no descriptores.

Una vez los términos se agrupen por estas cinco áreas temáticas nos centramos en la identificación de los sinónimos dentro de cada una. Detectadas las equivalencias semánticas procedimos a seleccionar el descriptor y a ubicar el resto dentro de los no descriptores. Se utilizaron las nomenclaturas USE y UP. Cada uno de estos datos serán incorporados en nuestras fichas. Por ejemplo,

donativos UP dádivas

dádivas USE donativos

Las notas de alcance para cada uno de los términos que lo demanden son muy útiles. Por ejemplo,

prefecto de estudios

NA Es el instrumento del Rector para ordenar los estudios y regir el seminario conforme a la facultad recibida.

Una vez seleccionados los descriptores y establecidas las notas de alcance necesarias, procedimos a establecer la jerarquía de los mismos, estableciendo los diferentes niveles jerárquicos, hasta la consecución de una estructura piramidal donde quedaran ubicados todos los descriptores. Las relaciones jerárquicas se manifiestan por medio de los operadores TG (Término Genérico) y TE (Término Específico). No se acepta en nuestro tesauro la multijerarquía, es decir, cada descriptor se asigna exclusivamente a un área temática. Por ejemplo,

conductas indebidas TG conductas seminaristas conductas seminaristas TE conductas indebidas

Además, los descriptores del mismo nivel jerárquico dependientes de un mismo término han sido ordenados de forma alfabética. Por ejemplo,

conductas indebidas
TE escándalos
TE rebeldía

Una vez creada la estructura jerárquica, nos centramos en el establecimiento de las relaciones asociativas entre descriptores; vinculando aquellos términos que, sin mantener una relación jerárquica directa, sí que mantienen una relación de tipo semántico (TR). Por otra parte, se han establecido cuatro tipos de restricciones. En primer lugar, no existen relaciones asociativas entre descriptores y no descriptores. Tampoco existen relaciones asociativas entre descriptores ya unidos por una relación jerárquica. Además, todas las relaciones son recíprocas; por ejemplo, conductas indebidas TR expulsión seminaristas expulsión seminaristas TR conductas indebidas

Finalmente, cuando el término se asocia a otro de nivel superior, se supone que está también relacionado con todos los subordinados a él. Por ejemplo,
patrimonio TR censos
Implica que,
Patrimonio
TR capellanías
TR diezmos
TR mandas pías
TR obras pías

Una vez acometidos los pasos expuestos, se está procediendo a la implantación de nuestro tesauro de forma provisional. Se están comprobando todos los pasos, incluyéndose nuevos términos pertinentes, eliminándose los descriptores demasiado específicos, e introduciendo otras modificaciones necesarias, dado que no es un instrumento estático y somos conscientes de que deberá ser revisado y ampliado constantemente: inclusión de nuevos términos; reducción de descriptores poco usados a no descriptores; inclusión de descriptores de un grupo jerárquico en otro; modificación de relaciones de equivalencia; e incorporación o eliminación de otras relaciones semánticas, etc.

El tesauro muestra el conjunto de términos en tres índices: alfabético, jerárquico y permutado Kwic.

En el índice alfabético aparecen todos los descriptores y no descriptores, con todas sus relaciones y notas de alcance. Con cada descriptor se presenta toda su jerarquía. Así cada descriptor consta de la indicación del descriptor genérico, descriptores específicos, descriptores asociados, nota de alcance, términos equivalentes, código del término. Por ejemplo,
diócesis - $\mathrm{xxxx}$ un prelado.
UP jurisdicción eclesiástica
TG circunscripciones
TE Badajoz (diócesis)
TE Madrid (diócesis)
TR obispados
TR obispos
TR visitas pastorales

NA distrito o territorio en el que ejerce jurisdicción

Para los no descriptores, únicamente se el descriptor principal. Por ejemplo,

jurisdicción eclesiástica USE diócesis

En el índice jerárquico aparecen únicamente los términos principales estructurados jerárquicamente. En él, podemos observar fácilmente la 
jerarquía establecida y la posición de cada uno de los términos dentro de ella. La representación de la cadena jerárquica se ha elaborado a través de sangrados y especificado mediante un sistema de codificación. Por ejemplo,

$$
\begin{aligned}
& \text { circunscripciones } \\
& \text { diócesis } \\
& \text { Badajoz (diócesis) } \\
& \text { Madrid (diócesis) }
\end{aligned}
$$

El índice permutado kwic muestra todos los términos para facilitar de este modo la búsqueda inicial y remitir así al usuario a la parte alfabética. Además, los descriptores van seguidos del código de identificación que remite al listado jerárquico y alfabético.

En resumen, la utilidad fundamental del tesauro diseñado para el ASMMB es que nuestros usuarios puedan consultarlo con facilidad. Normalmente formularán su búsqueda en un lenguaje ordinario para después elegir los descriptores, dándose tres situaciones:

1. Que la consulta haya sido formulada con precisión y los conceptos figuren en el tesauro. Para ello el usuario consultará en primer lugar el índice alfabético del tesauro donde encontrará los descriptores que corresponden a su consulta. Por ejemplo, para la consulta "Expedientes personales del Seminario San Atón de Badajoz del año 1857", los descriptores son expedientes personales AND Badajoz AND Seminario San Atón.

2. Que la consulta sea precisa, pero el tesauro no incluya los conceptos. En este caso después de la consulta del índice alfabético y kwic, el usuario accederá a la parte jerárquica y buscará allí los conceptos que más se asemejen. Por ejemplo, para la consulta "Admisiones de alumnos sin recursos en el Seminario de Badajoz", los descriptores son admisiones AND becas AND Badajoz AND Seminario San Atón.

3. Que la consulta no sea precisa. El usuario accederá a la parte jerárquica y elegirá allí los descriptores más adecuados. Por ejemplo, para la consulta "ceremoniales de órdenes" deberá buscar a través de Órdenes Sagradas.

\subsection{Gestor documental}

Llegamos al tercer elemento del sistema de información del ASMMB. Por medio de esta herramienta, elaborada gracias al empleo de tecnologías de la información, es posible acceder la información relativa a cada documento de manera sencilla y rápida, tanto a las características externas como al contenido de los docu- mentos a través de un interfaz muy cercano al usuario, cuestión primordial si tenemos en cuenta que nuestros principales usuarios, sacerdotes y seminaristas, desconocen la gran mayoría de las cuestiones anteriormente expuestas y buscan la sencillez y agilidad en la recuperación de los documentos que solicitan. Los objetivos que pretende cumplir esta herramienta son: elaborar un instrumento automatizado que nos permita la consulta de los documentos por medio de un intefaz cercano al usuario; diseñar un sistema que nos facilite realizar consultas con el fin de localizar y recuperar la documentación del archivo; y elaborar un sistema de información automatizado que proporcione las características externas e internas de cada documento. En base a ello, el gestor documental que hemos diseñado para el ASMMB presenta un interfaz muy sencillo, por medio de ventanas y botones, para que nuestros usuarios puedan manejarlo sin ningún tipo de problemas y recuperar la documentación pertinente con rapidez. A continuación se presenta su estructura básica.

La ventana inicial presenta una estructura elemental que identifica a la entidad y a su archivo (logo) y que ofrece tres botones: mantenimiento, consulta y salida.

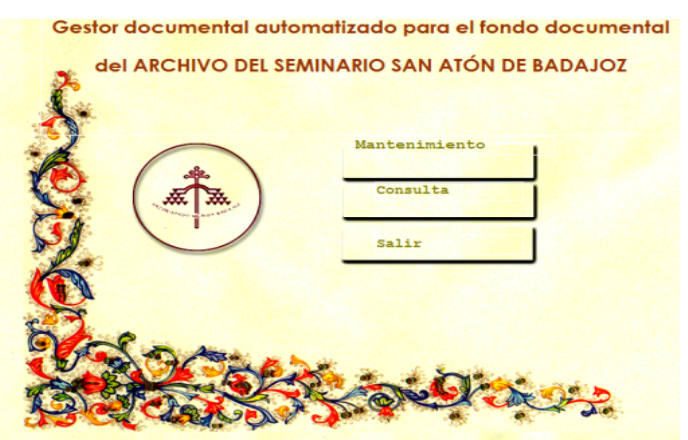

Figura 1. Ventana inicial de gestor documental

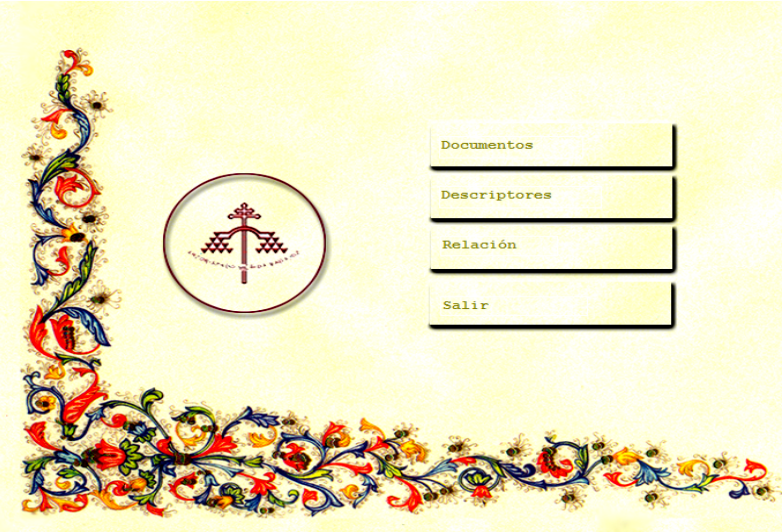

Figura 2. Pantalla de mantemiento 
Aunque en principio se parte de la base de que el total de documentos custodiados en el ASMMB se han de ofrecer en el catálogo; sin embargo, somos conscientes de que con el paso del tiempo deberán ser añadidos nuevos documentos, al tratarse el Seminario de una entidad viva en la que se siguen desarrollándose tareas educativas de rango universitario. Por ello, se ha creado esta primera opción, que nos permite también la inclusión de datos, a la que se accede mediante el botón "Mantenimiento".

A través de él se accede a una pantalla que ofrece cuatro funciones. El botón "Documentos" permite la incorporación de un documento completo o la modificación de alguno de los ya registrados. El botón descriptores: se utilizará para la incorporación de nuevos descriptores: geográficos, onomásticos, topográficos y de materia. El botón "Relación" permite la modificación de los descriptores existentes, establecer relaciones entre los descriptores y los documentos de nueva incorporación y modificar las relaciones establecidas entre documentos y descriptores. Finalmente, el botón salir devuelve a la pantalla inicial.

La segunda opción de la pantalla inicial es el botón "Consulta" (Figura 3). Por medio de la pantalla correspondiente a esta opción podremos acceder a la documentación que ya se encuentra incluida en la base de datos para posteriormente recuperarla. Permite la búsqueda libre, por descriptores, a través del tesauro y mediante los índices.

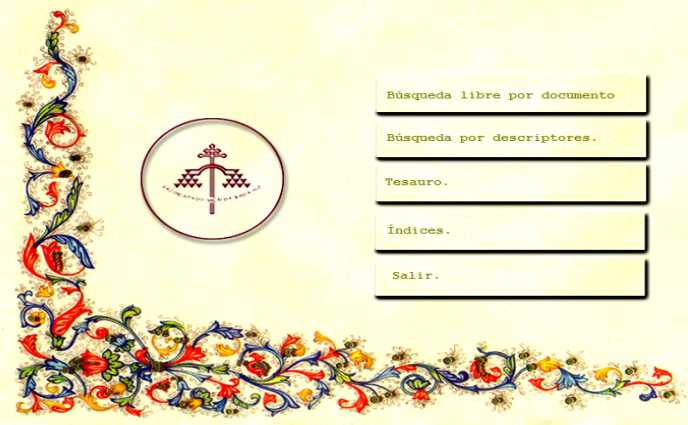

Figura 3. Pantalla de consulta

La "Búsqueda libre por documento" permite la localización de los documentos a partir de términos y cadenas de cualquiera de los campos que constituyen la ficha documental.

La "Búsqueda por descriptores" es la opción clave de este gestor, puesto que nos permite la localización de los documentos a través de la combinación de los descriptores que han sido asociados a los mismos y de los operadores lógicos normales en este tipo de sistemas $(\mathrm{Y}, \mathrm{O}$, NO).

El botón "Tesauro" permite de forma automatizada el acceso al tesauro presentado en el epígrafe anterior en sus diferentes presentaciones (Figura 5).

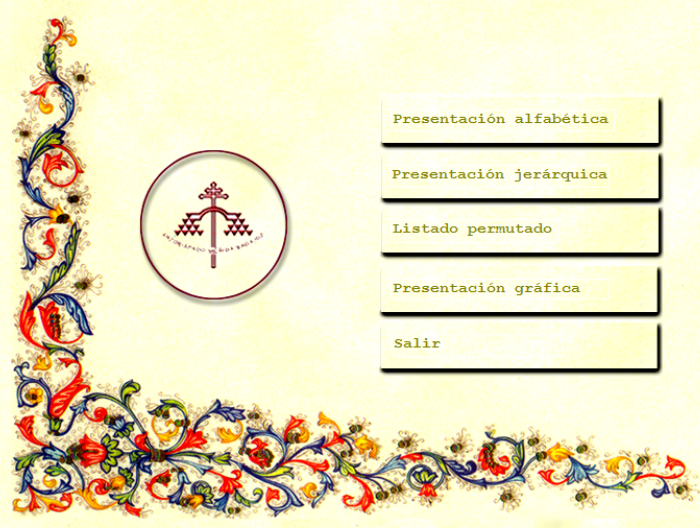

Figura 5. Ventana del tesauro

Finalmente, el botón "Índices" conduce a la ventana de consulta relativa a los índices. En ella podemos acceder a la información contenida en los seis índices previstos (Figura 6).

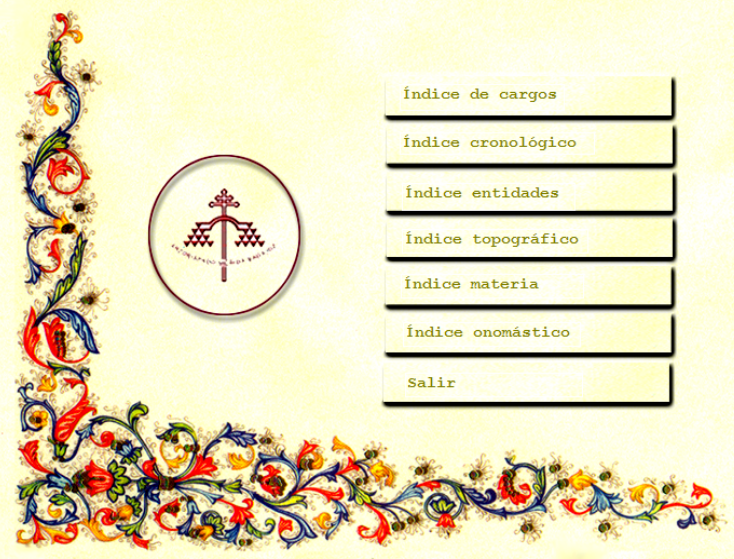

Figura 6. Ventana de acceso a los índices

Una de las tareas pendientes a ejecutar en el gestor documental es la incorporación de los documentos digitalizados. Somos conscientes de la utilidad de esta función máxime para aquella documentación muy deteriorada. El diseño del sistema prevé la digitalización, aunque el proceso es arduo, dado el amplísimo volumen de documentos y la falta de recursos, especial- 
mente falta de personal que permita desarrollar con celeridad esta cuestión.

\section{Conclusiones}

Los archivos de la Iglesia conservan una importantísima parte de la cultura de todos los tiempos. En especial, los archivos de los seminarios custodian la documentación producida y recibida por estas entidades en sus actividades educativas y pastorales. Por tanto, debemos tener en estos archivos un referente clave a la hora de emprender estudios relacionados con la educación. En el caso que nos ocupa, el Seminario San Atón de Badajoz guarda documentos desde el siglo XV intrínsecamente relacionados con sus actividades educativas y pastorales. Aquellos que acometan investigaciones sobre el panorama educativo de la sociedad extremeña desde el siglo XVI tienen en el ASMMB documentación única que aportará a sus trabajos cimientos de suma relevancia para la consecución satisfactoria de los mismos.

Es necesario remarcar la necesidad urgente de que en todos los archivos de la Iglesia se efectúen tareas de ordenación y clasificación de sus fondos que, no sólo saquen a la luz documentación hasta la fecha inédita, sino que eviten su destrucción, es decir, la lapidación de la historia eclesiástica española. El Seminario de san Atón, consciente de este hecho, trabaja en salvaguardar su patrimonio; y ejecuta tareas que van a permitir poner al servicio de la sociedad la documentación de sus archivos.

La gran aportación de este trabajo es la creación de un Sistema Integral de Información Histórica para la documentación del Seminario
Metropolitano San Atón de Badajoz desde el siglo XV hasta nuestros días, integrado por tres pilares -catálogo, tesauro y gestor-, que tienen como finalidad principal la recuperación y difusión de la documentación contenida en este archivo. Como resultado del trabajo realizado, se está poniendo al servicio de la comunidad científica extremeña y del propio Seminario una documentación que se encontraban aislada, archivística e históricamente.

\section{Referencias}

Heredia, Antonia (1982). Manual de instrumentos de descripción documental. Sevilla: Diputación provincial, 1982.

ISAD (G). General International Standard Archival Description (2000). $2^{\circ}$ ed. Ottawa: ICA, 2000.

López Yepes José (ed.) (2004). Diccionario Enciclopédico de Ciencias de la Documentación. Madrid: Síntesis, DL. 2004.

UNE-ISO 25964-1:2014. Tesauros e interoperabilidad con otros vocabularios. Parte 1: Tesauros para la recuperación de la información. Madrid: AENOR, 2014.

Pérez Ortiz, Guadalupe; González Lozano, Francisco (2014). El archivo del Seminario Metropolitano San Atón de Badajoz: organización de sus fondos documentales. // Revista Ibersid. 8 (2014) 55-66.

Rubio Merino, Pedro (1999). Archivística Eclesiástica: Nociones básicas. Sevilla: Guadalquivir, 1999, 328.

Slype, G.V. (1999). Lenguajes de indización: concepción, construcción y utilización en los sistemas documentales. Madrid: Fundación Germán Sánchez Ruipérez, 1999.

Valle Gastaminza, Félix del (1991). La aportación de la Documentación a la descripción en un medio de archivos. // IRARGI IV (1991) 108.

Enviado: 2015-02-26. Segunda versión: 2015-06-10. Aceptado: 2015-06-16. 